\title{
AN OVERVIEW OF TECHNOLOGICAL ADVANCEMENTS AND FUTURE POSSIBILITIES IN WIRELESS POWER TRANSMISSION
}

\author{
Tanmay Sawant ${ }^{1}$, Durvesh Pilankar ${ }^{2}$, Radhika Sule $^{3}$, Shweta Mahadeshwar ${ }^{4}$ \\ ${ }^{1,3}$ Student, Department of Electronics and Telecommunication, St. Francis Institute of Technology, Maharashtra, India, \\ ${ }^{2,4}$ Student, Department of Electronics and Telecommunication Vidyavardhini College of Technology, Maharashtra, \\ India,tanmaysawant092@gmail.com,durveshpilankar92@gmail.com,radhikasule24@gmail.com, \\ shweta22393@gmail.com
}

\begin{abstract}
Electricity has been one of the major factors for various popular inventions in world's history. Most of the developments in today's world would have been impossible without the existence of electricity. The main mode of transmission of this electrical energy available to all of us is through wires but efficiency is significantly reduced in power transmission through wires. Only $71 \%$ of the electrical energy can be transferred efficiently. Moreover it is always difficult to lay cables in remote area. All these factors have given rise to necessity of a concept where the losses are minimized and convenience of energy transfer is increased. The concept being wirelessly transmitting power i.e. WITRICITY. In this paper we have given an overview of the recent researches and advancement in the field of wireless transmission of electricity. Various methods of wireless power transfer such as laser beaming, microwave power transfer, solar and magnetic resonant induction coupling are explained. We have also discussed the future aspects of wireless power transmission and have stated its various advantages. This paper helps us in understanding thoroughly the alternative method of transmission of power.
\end{abstract}

Index Terms: Wireless Power, WiTricity, Magnetic Resonance Coupling

\section{INTRODUCTION}

Today we live in an era of Wireless Technology where we use various advanced devices like mobiles,mp3 players, laptops, home robots etc. These devices no matter how much advanced, sophisticated and portable consist of batteries that eventually gets exhausted. And to charge these devices we need a wired charger or wired connection. Though in this $21^{\text {st }}$ century we may have reached MARS but still we have not been able to eliminate the wired connection totally.

Nowadays data or information can be conveniently transmitted from one part of the world to other part through wireless connection but transmission of electrical energy has been still confined to wires!! WiTricity has its roots based on the fundamentals of physics mostly related to electromagnetism. Back in the 1890's when it was decided that electricity should be made available to every household, NIKOLA TESLA one of the great pioneers of electromagnetism never thought that people would actually drag wires around the entire globe to use electricity. He had always dreamt of transferring electrical energy wirelessly to distant places. This would have eliminated the cost of cables that had to be laid throughout the area for transmission of electricity and had many other advantages too!! Apart from that wireless power transmission can be easily used to supply power where wired power transmission was very difficult to reach, example: hilly regions, remote islands etc. Tesla also gave a theoretical explanation by how it could be achieved by using big electromagnetic resonators. An electromagnetic resonator generates very large electric field. This can be propagated by conduction through ionosphere through gigantic sparks or through the earth via intermediate coupling to earths charge resonance also known as Schumann Resonance[1]

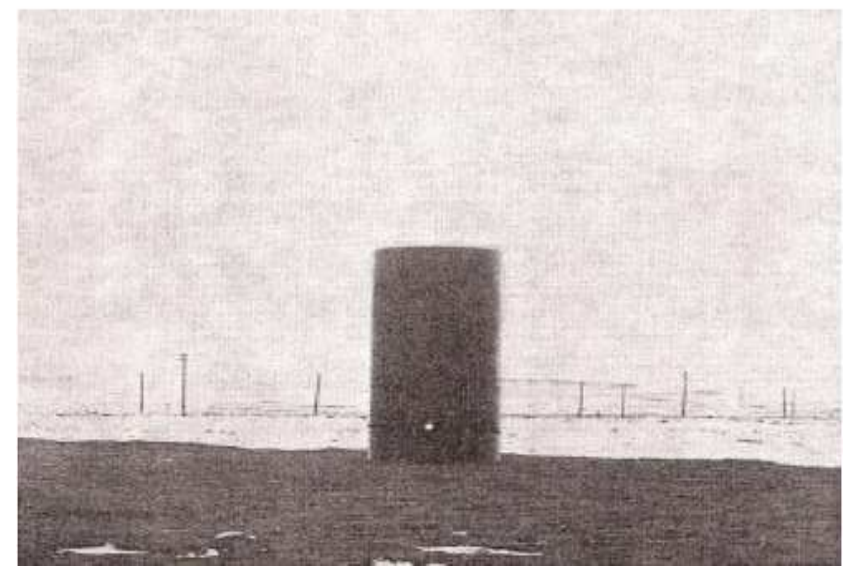

Fig-1: Tesla's Colorado Spring experiment 
The above picture shows that it's a experiment conducted by Tesla at Colarado Springs wherein he had illuminated a bulb in the bottom part of the picture [2]

Tesla further began to work on his dream and his epitome was the Wardenclyffe Tower[3]. It was a $57 \mathrm{~m}$ high structure which was meant for transmission of electrical energy to the whole world i.e. it was meant for trans-atlantic telephony and wireless broadcasting purpose.

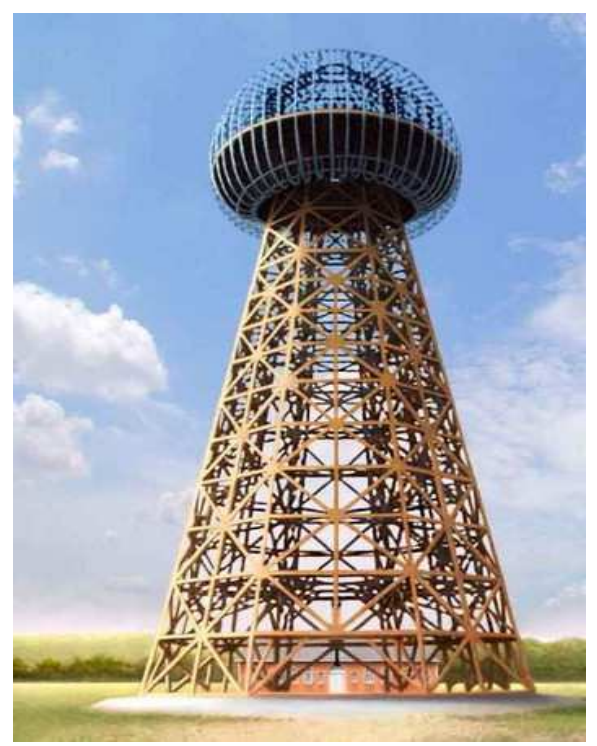

Fig2: Wardenclyffe Tower

Later it was demolished because it was hardly operational. But it was Nikola tesla who gave the first hope that wireless electricity could replace the lossy power cables.

\section{TEAM MIT REVISITS WIRLESS POWER:}

Although Tesla had invented the wireless power nearly 100 years back, but what made MIT team revisit this promising technology is when Prof. Soljačić, who many times used to forget to keep his phone on charge during night and one night he happened to wake up due to the battery low sound that his phone made and thus as you would guess this spurred him to think that wouldn't it be great if phone could charge by itself!!

Thus led by Prof. Soljačić ,team from MIT's Department of Physics, Department of Electrical Engineering and Computer Science, and Institute for Soldier Nanotechnologies (ISN) has indeed followed the footsteps of Tesla. They acknowledged Tesla's theory of Wireless Electricity which is implemented by Tesla Coil[4] and moreover this team put forth a theory that there is a way wherein they could make the Wireless power transfer non radiative and more prominent even when the distance between the two coils could be extended by a few feet by adding magnetic resonance into the picture and achieving better efficiency

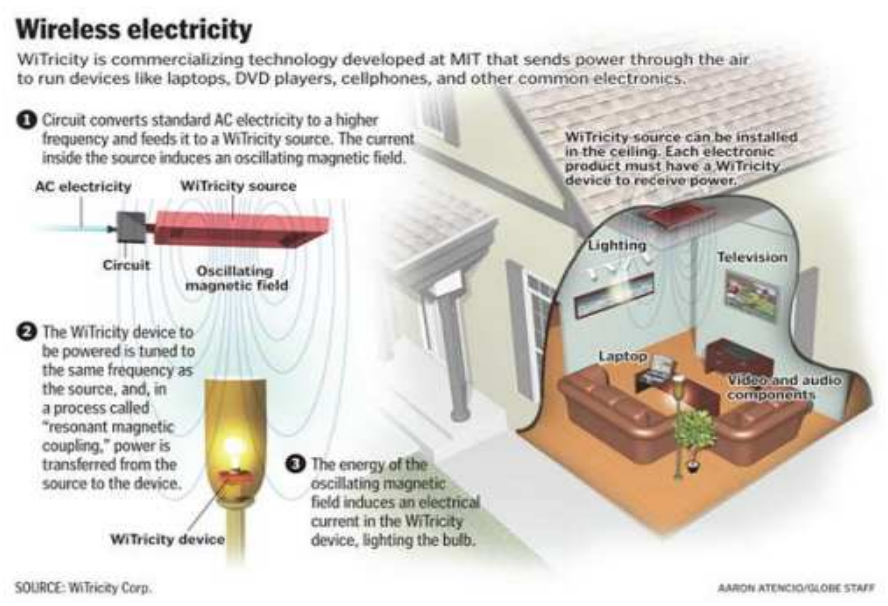

Fig-3: WiTricity diagram for Wireless Power

They demonstrated the concept of 'WiTricity'[5] which meant that they could illuminate a light bulb of 60 Watt which was practically more or less 2 meters away from the power source albeit there was no connection between the power source and the light bulb.

\section{INTEL'S EXPMERIMENT}

Intel in 2008 demonstrated the application of Wireless Power that practically has a strong potential to get rid of chargers, batteries or power chords.

Intel presented at Intel Developer Forum wherein they powered a 60Watt globe at a distance of 3 feet away from the resonator with almost $75 \%$ efficiency. This technology is based on Resonant Magnetic Induction. It is the same technology used by the MIT team too. Importantly, they managed to transmit power without any trouble even though some obstacles were made to pass through the sending and receiving units. They also maintained that these magnetic fields which form the core principle of this technology are not harmful to humans or animals, only the electric field which can be proved harmful is not used in this technology.

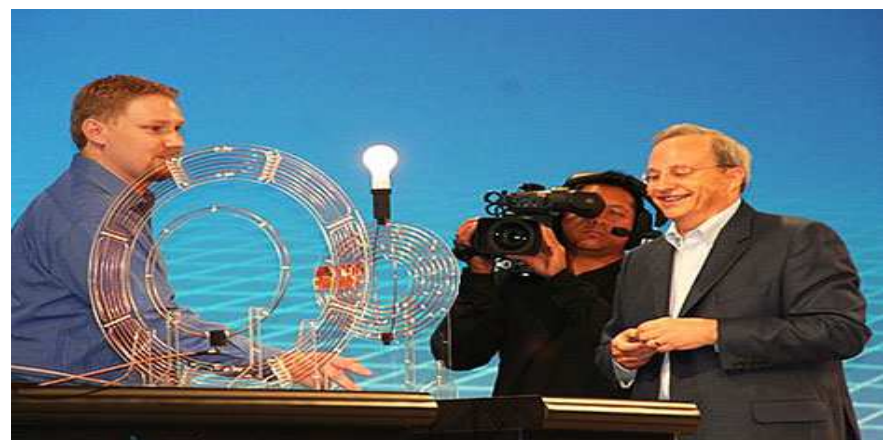

Fig-4: Intel demonstrating their Wireless Power Tech at San Francisco 
Intel believes that they could well use this Wireless power technology on a wider scale including home use such as to power laptops and other portable electronic devices. Now to carry out further research and bring this technology into real use Intel has partnered with IDT( Integrated Device Technology, Inc).Apart from this just recently in June 2013,Intel has joined the 'Alliance for Wireless Power (A4WP)'[6] which has been jointly established by Samsung and Qualcomm to serve the same purpose

\section{KOREAS INNOVATION}

In Aug 2013, Koreans have certainly picked up on this technology and have actually implemented Wireless electricity effectively.[7]

Since due to the depleting fuel reserves, the need of an hour has led to the demand and production of electric vehicles. But the main drawback of these Electric vehicles was that they had to be stopped to get recharged in a particular location whenever their batteries ran low.

This was getting increasingly inconvenient so the Koreans devised a technique making use of the already implemented Wireless Electricity. The Team of Korean Engineers have developed a long $7.5 \mathrm{~m}$ hi-tech road wherein the recharging system which is designed by Korea Advanced Institute of Science and Technology was installed.

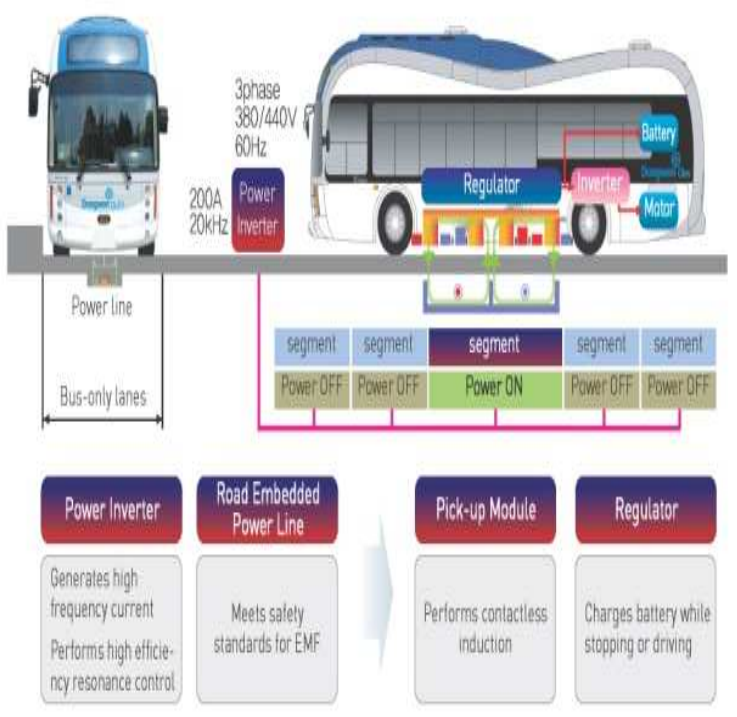

Fig-5 A schematic of Wireless Power used in Korean Bus

The system involves devices which are installed beneath each of the bus and there are electric cables which generate electromagnetic waves are fitted in specific parts of the road which ultimately covers the entire range of $7.5 \mathrm{~m}$. Thus if this technology is implemented on a large scale, then well this would really revolutionize the automotive world

\section{TIMELINE:}

1864: James Maxwell conducts observations of electricity, magnetism and models the behavior of Electromagnetic Radiation and comes with what is known as "Maxwell equations"

1888 : Confirmation of existence of Electromagnetic Radiation by Heinrich Rudolph Hertz through " apparatus for generating electromagnetic waves" was a VHF or UHF "radio wave" spark gap transmitter."

1893: Tesla's Demonstration of Wireless power transfer through wireless illumination of phosphorus lamps in Chicago 1896-97 : Tesla proves wireless transmission over a distance of $48 \mathrm{~km}$ and files his patent on Wireless Transmission

1902: Tesla vs. Reginald Fessenden - U.S. Patent Interference No. 21,701, System of Signaling (wireless); selective illumination of incandescent lamps, time and frequency domain spread spectrum telecommunications, electronic logic gates in general.

\section{7: Wardenclyffe tower of Tesla demolished}

1961: Brown shows the possibilities of microwave power transmission by publishing a paper

2000: Using "vertical flux" approach and resonant power transfer for charging portable electronics, Prof Shu Yeun (Ron) Hui invented a planar wireless charging pad.

2005: Prof. Shu Yuen (Ron) Hui and Dr. W.C. Ho do research on a planar wireless charging platform having freepositioning feature. On a flat surface, the pad is able to charge several loads. Publishes the paper in IEEE Transactions

2007 : Using Magnetic Resonance, WiTricity Team from MIT led by Prof Marin Soljacic power a $60 \mathrm{~W}$ bulb wirelessly 2 metres $(6.6 \mathrm{ft})$ distance with two $60 \mathrm{~cm}$-diameter coils

2009: Palm Pre phone launched with Palm Touchstone wireless charger.

2009: Sony demonstrates a wireless electro dynamicinduction powered TV set, $60 \mathrm{~W}$ over $50 \mathrm{~cm}$

2013: The concept of a virtual waveguide controlled by ordered magnetic fields for wireless power transmission is proposed. 


\section{BASIC TERMS RELATED TO WITRICITY}

\subsection{Magnetic Induction:}

Magnetic flux, in simple terms is any area that has a magnetic field passing through it. Changing magnetic flux induces an electric current in conductor in vicinity of it. Magnetic flux can be altered either by varying the strength of magnetic field or by motion of conductor through the magnetic field. Such generation of an electric current in a conductor is called as Magnetic Induction. Some examples of devices based on magnetic induction are electric transformers and electric generators.

When alternating current is passed through a loop of conducting material, it generates oscillating magnetic field in the vicinity of it. Oscillating magnetic field indicates change in the strength of magnetic field. When another conducting loop is brought closer to the first one, it captures some portion of this oscillating magnetic field. Thus electric current is induced in it which can be used to power devices. Electric transformers works on this principle

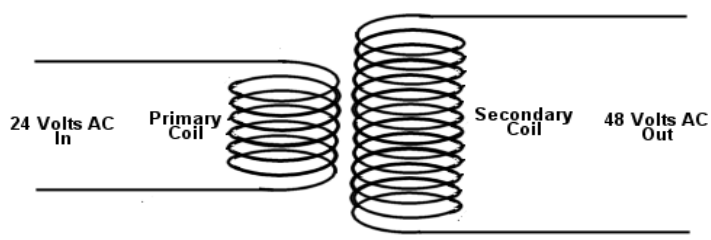

Fig-6: Magnetic Induction between Primary and Secondary Coils

In case of electric generators, wire loop inside the generator is mechanically driven by some source of rotary motion. This wire loop spins in a magnetic field. Thus electric is current produced in the wire.

The principle of magnetic induction can be used for the transfer of electrical power without any physical contact in between.

\subsection{Energy Coupling:}

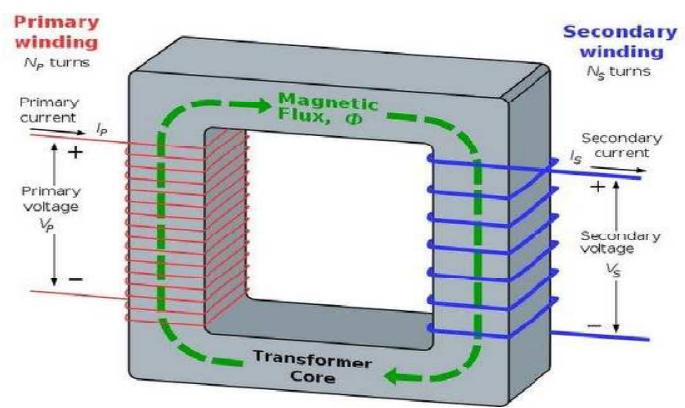

Fig-7 Energy coupling between primary and secondary windings
Coupling basically means interaction between two devices or circuits. Energy coupling is a phenomenon in which electrical energy is transferred from one device to another. Transformers are example of couplers. Magnetic coupling is said to occur when the interaction between the couplers is due to the magnetic field of one of the couplers. No contact in form of wires or connectors is required for magnetic coupling. The magnetic field of one of the body induces current in the other body of the coupling system. This way power can be transferred from sending unit to receiving unit. An electric transformer can be used for this purpose, in which AC current is transformed from one voltage to other

\subsection{Electrical Conduction:}

In this electrical energy is transferred through the upper atmosphere due to ionization of the gas. It also includes transfer through naturally present conductors. Naturally present medium example water, air can be made conductive. This can be done by going beyond the breakdown voltage and ionizing the gases present in it.

\subsection{Electro-dynamic Induction:}

In this electrical energy is transferred from one circuit to another by the principle of induction[8]. In this the distance between is coils must small. The receiving unit will pick up the transmitted energy efficiently when the frequency is matched. Use of resonators also increases the efficiency. The receiving and sending units must be adjacent to each to avoid losses. To further improve the performance a non sinusoidal wave is transmitted. The coils used are solenoids with capacitor, this helps to tune the receiver frequency to that of the transmitter.

\subsection{Electrostatic Induction:}

It is also known as capacitive coupling. It is the differential capacitance between two electrodes which are used for wireless transmission. In this high frequency A.C potential is transferred from one component to other. Thus energy is transferred from sending unit to receiving unit.

\section{METHODS OF TRANSMISSION}

\subsection{Laser Beaming}

A. Laser Beaming-.in the process of LASER beaming, transfer of power is in the form of laser. The energy received at the receiver is then converted into electrical energy. The first test flight of an aircraft powered by a laser beam was conducted inside the building of NASA Marshall Space Flight Center. It is also stated as power beaming because the power is beamed at receiver that can further be converted to electrical energy for our use

One of the major disadvantage is that the laser beaming can be hazardous to human health. It also requires direct line of sight. 
If direct line of sight is not provided then there is a loss of energy in the absorption process.

\subsection{Microwave Power Transfer}

Microwave transfer basically is the transfer of energy using radio waves which are having wavelengths measured in centimeters generally referred to as microwaves. Transfer of electricity wirelessly using radio is possible by using Rectenna for conversion of the transmitted radio waves into electricity. Rectenna with efficiencies up to $95 \%$ have been developed for this purpose.

Rectenna is basically a combination of Rectifier and an antenna. This technique known as Microwave power transmission (MPT) is primarily useful for transmitting power energy from the solar power satellites to Earth obviously without using wires. William $\mathrm{C}$ Brown also demonstrated flying a miniature helicopter making use of the device rectenna
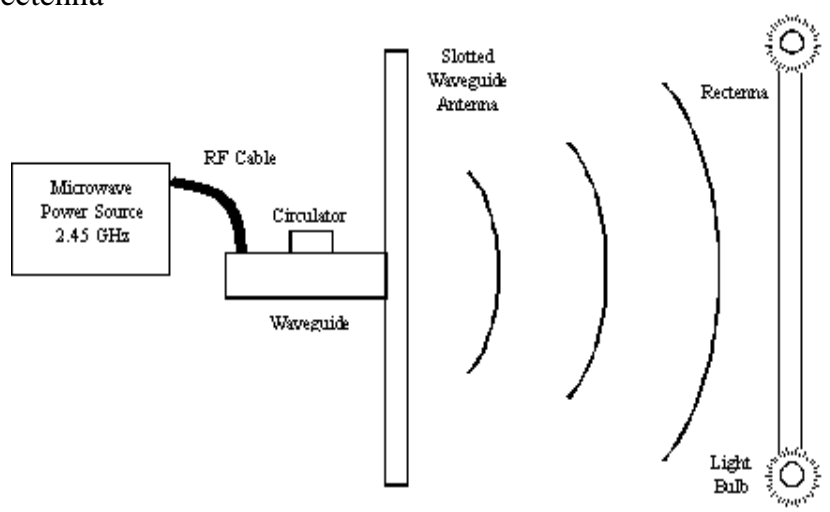

Fig-8: Microwave Power Transmission

The figure shows exactly how the Microwave Power Transmission works[9]

Though lasers as explained above can also be used, but their low efficiency in light generation and reception has deterred the use of it and has favored the use of MPT.

There have been concerns over the use of Microwave Power Transmission but it has been proved safe as it is suggested that power levels would be more or less similar to that of the leakage from an microwave Owen and a cell phone.

\subsection{Solar}

Solar power also can be considered as the concept of wireless energy. Every day we receive large amount of energy from the sun through solar panels. This solar energy is further converted to electrical energy by using a transducer.. Furthermore Research is going on to bring about an improvement in the efficiency of the power by using the solar panels.
By using the solar power concept we can utilize the energy up to $100 \%$ efficiency. Cost still remains the major concern in this method but solar gives us clean electric power which can be used throughout the globe. In the coming future satellites may be solar powered and the may be providing energy to Earth by wireless power transmission

\section{WiTricity}

WiTricity is nowadays the latest and the most trending technology when we consider wireless power transmission. The based fundamental used in this technology is the magnetic resonant coupling. This is because two resonant objects of the same resonant frequency tend to exchange energy more efficiently to a considerable amount of distance.

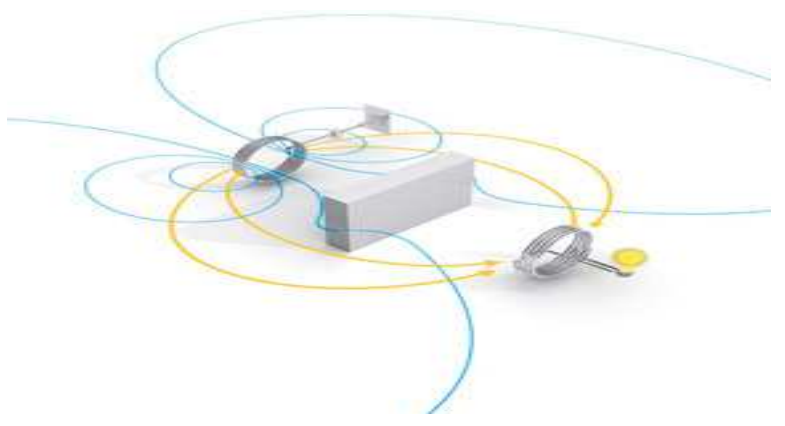

Fig-9: WiTricity illustration

WiTricity power sources are actually devices that are specially designed magnetic resonators. These magnetic resonators can be used for efficiently transferring power over distances via the magnetic near-field.

In the above figure of WiTricity power source, the left side is connected to AC power or we can call it the primary side. The blue lines represent the magnetic near field induced by the power source because of the primary connection. The yellow lines represent the flow of energy from the source to the WiTricity capture coil or the secondary coil. We can see that the bulb glows as the current is induced in the secondary coil which is the result of magnetic resonant induction coupling.

\subsection{Magnetic Resonant Induction Coupling used in WiTricity}

Magnetic Resonant Induction Coupling forms the basis of WiTricity. This basically involves two bodies which exchange energy by magnetic oscillations which is much more efficient than wired exchange.

Inductive coupling is when two conductors are magnetically coupled. In this current through one wire induces voltage in the other wire. This is caused due to electromagnetic induction. When the two conductors or coils are adjusted so that they resonate at the same frequency the phenomena is 
called Resonant Induction Coupling. Due to the resonance a strong interaction is set up between the sending unit and the receiving unit. As per the work of MIT scientists, when a pair of electromagnetic resonators is coupled using magnetic field a strong connection is a observed. Using this phenomena power transfer from one resonator to other is possible.

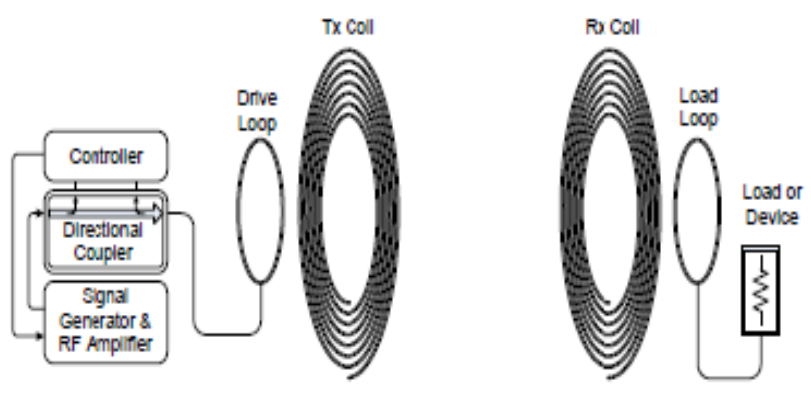

Fig-10 : Magnetic Resonance Induction Coupling

The distance between the pair of resonators being much larger than the resonator itself. The coil connected to a power source acts as the sending unit. The function of this coil is to emit non radiative magnetic field. The magnetic field is in the range of mega hertz. The receiving coil resonates with this magnetic field and a connection is established between sending and receiving coils. The magnetic field establishes a strong interaction between the resonators. The interactions with the surrounding environment are weak and hence magnetic coupling proves to be efficient for WiTricity. Thus power can be transferred over long distances without being affected by external environment. It can also be possible to have a single sending unit and multiple receiving units. These can be located at remote places and connected to a single central sending unit.

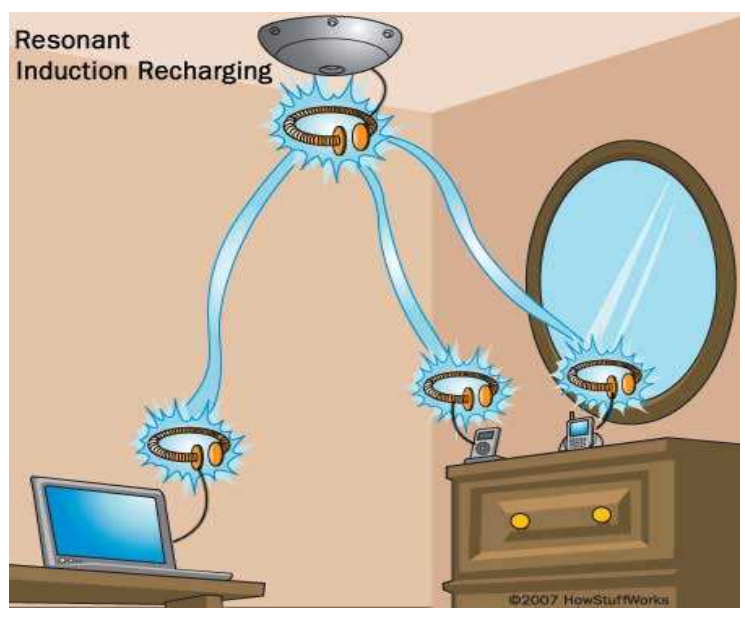

Fig-11 Resonant induction coupling between 2 coils which can charge devices like phone and laptops

\section{FIELDS OF TRANSMISSION:}

\subsection{Near field theory}

Near field principle of transmission is usually magnetic energy transfer, in which the distance is comparable to one-fourth of the wavelength to be transmitted. In this phenomenon resistive loss is observed.

\subsection{Far field theory}

Far field is also known as radiation zone. This theory is for long range distances, where the distance to be covered is much larger than the device. Directivity is a major concern in this phenomenon which is affected by diffraction. Increasing the directivity increases the efficiency. Using high directivity or a advanced laser can increase the efficiency by large.

\section{FUTURE ASPECTS IN WIRELESS POWER}

\subsection{Energy saving in WSN by using WITRICITY}

Wireless sensor networks (WSN) are also one of the most trending and emerging technology nowadays. It consist of numerous number of nodes distributed over a particular area of our interest. Every node consists of 4 components-sensor, data processing unit, transreceiver and most importantly a battery to supply the other three components with required power. The sensor first senses the data which is further processed by the data processing unit e.g micro-controller etc. The processed data is further transmitted to the base station by the transreceiver. There are many different parameters which reflect the efficiency of the WSN. One of the most important parameters of WSN is Energy saving in batteries. This is because most of the sensor nodes in the network are placed at remote areas for environmental and military application. These sensor nodes should be very small in size so as to reduce the cost $n$ to make it more portable and advanced.

Because of this the battery attached to the node is very small too. This small battery has to be used for different processes like sensing, data processing and transmission. After a particular interval of time these batteries may get completely exhausted and hence data in that region will never reach the base station. Hence it becomes really important to save the energy of the battery as one cannot replace it from time to time. Also wired charging of these batteries is almost impossible too as they are placed in remote area.

The concept of WiTricity can be used here as it can be easily used to transmit power to the remote areas. Various primary stations can be constructed and these stations would have a particular area under their range. There may be large number of sensor nodes under this particular area. Consider a scenario that we have a field wherein we are sensing Water Level and as well as we are sensing Temperature variations and we have installed around 1000 nodes each node placed a few several feet away from each other. 
Now we will install a huge primary coil which will be large enough to cover almost 50 sensor nodes and then we can install 6-7 secondary coils which can provide wireless power to 50 nodes as and when the battery decreases thus increasing the life and efficiency of the node to its maximum. Similarly we can extend the process and cover the entire geographical area. Huge coils with strong magnetic resonance will prove to be extremely useful for this purpose.

\subsection{Third-Generation Wireless Power}

One of the most latest development in the field of WiTricity has been brought by PowerbyProxi[10]. It has developed a 3G wireless power delivery system which is much more efficient and powerful than the earlier systems. Previously wireless power technology were based on split transformers. It consisted of two halves: an input side which basically consisted the primary coil and an output side which had the secondary coil.

First electrical energy is applied to the primary coil. Now this energy is converted to an electromagnetic field that induces a current in the secondary. From the secondary this energy is passed on to a load which is at a distance nearer to primary coil. But in this kind of system efficiency was very low ie below $50 \%$ approximately and because of this reason practical implementation on a large scale was never done. PowerbyProxi system offers high efficiency levels compared to the earlier generation of wireless delivery systems..

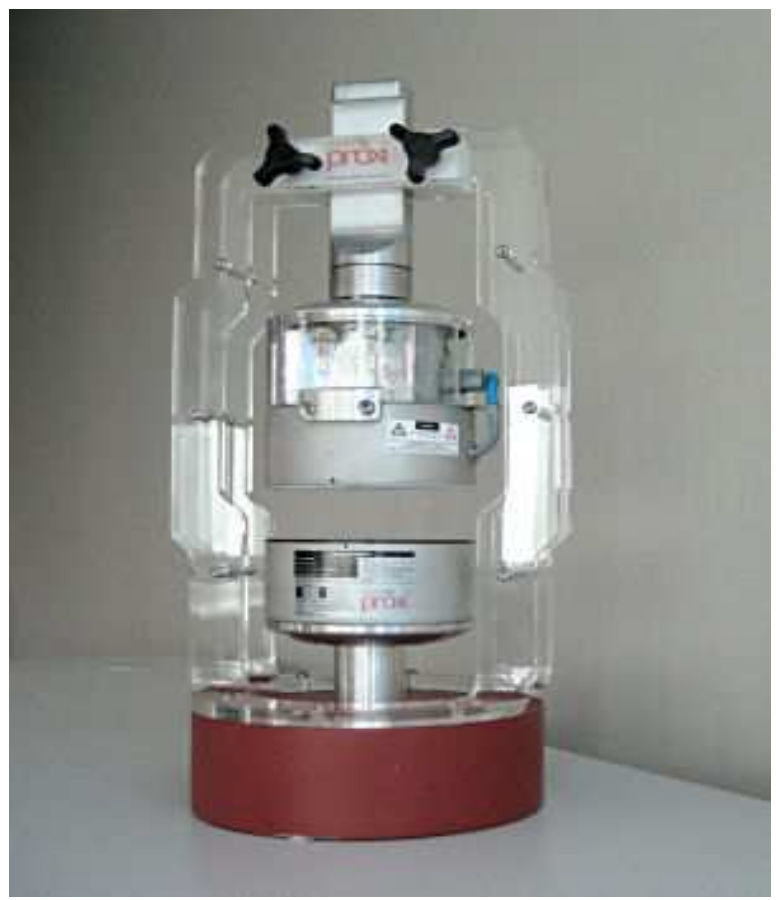

Fig-12: A demonstration model of the PowerbyProxi technology
PowerbyProxi uses coils to transmit and receive power between a power transmitter (PTx) and power receiver (PRx). These coils are analogous to antennas used for radio communication. Depending on their specific power requirements of the electrical devices, this power controller regulates the power flow to the device from the receiving PRx coil. Furthermore RF antennas need to be tuned to maximize the signal strength and increase efficiency and hence in PowerbyProxi a patented tuning technology called dynamic harmonization control (DHC) is used. It provides better performance by dynamically varying the frequency in response to environmental and load changes. Also various advantages like better efficiency, reduced receiver size \& negligible electromagnetic interference is achieved. Efficiency of around $90 \%$ can be achieved by using ProxiWave technology

\subsection{Power-Generating Solar Satellite Inhabitant}

One of the interesting is wirelessly transmitting energy from space to EARTH. Japan has already shown keen interest in this novel idea and it is planning to power up 3 million houses from this power.

They have serious plans to send a solar powered satellite[11] in space which will harness the energy of the sun. This harnessed energy will be sent to earth wirelessly through a beam that would be almost giga-watt strong

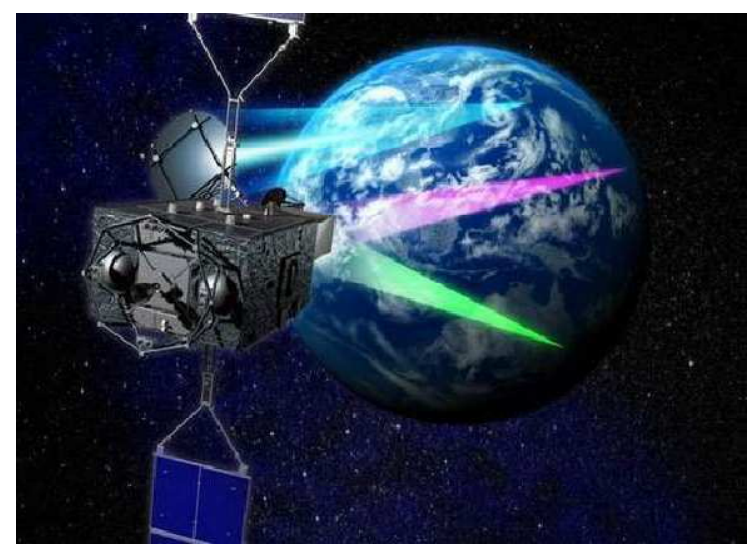

Fig-13: Japan's Wireless Power Transmitting Satellite

A test model will be launched by 2015 but to remove all the kinks and the flaws ,but it may take more than 3decades for this setup to be actually implemented. One of the most possible danger will be to transmit a giga watt microwave beam to a small spot on Earth and more than that to calculate the consequences if it misses the target.

\subsection{Architectural Point of View :}

Usage of Wireless power would also solve many problems related to construction of buildings. 
The duct space which contains the electricity wires which consumes a lot of portion will no longer be needed. Hiding Primary coil in the ceiling or under the floor will serve the purpose.

Also most of the causes for fires in the buildings are due to Electricity wires getting short circuited. Wireless Power would solve this issue with ease.

\section{ADVANTAGES OF WIRELESS POWER}

1-The main advantage is it is omnidirectional means the electrical energy generated can be transmitted in all the desired directions.

2-WiTricity replaces the use of power cables and batteries thus Installation cost reduces and we get cheaper electricity

3-More Convenient and Reliable

4-More Environmentally Friendly. It eliminates mess of cords, reduces toxic battery waste, reduces use of plastics ,rubber and metals.

5-Less accidents and instances of electrocution

6-It could eliminate the use of all the usage of batteries.

7-High transmission integrity and very low loss in its Transmission

8-Energy Transfer via Magnetic Near Field is non-radiative mode of transfer. Magnetic field tend to interact very weakly with the biological tissues of the body and so are not prone to cause any damage to any living beings.

\section{DISADVANTAGES OF WIRELESS POWER}

1. Currently the system is like the efficiency decreases as distance between source and capturing device increases.

2. Currently this technology is limited to small home appliances.

\section{CONCLUSIONS}

Wireless transmission of energy is a technique that holds a strong potential. It promises to change the face of the technologies in transmission of power. Its applications can be huge. In future there are possibilities that power can be transferred over large distances without affecting the environment. Using varies theories described above; electrical energy can be transferred to remote terrains. Another important issue being use of batteries can be reduced; replacement and recharging batteries would not be required. Use of wireless technology also saves on the resources spent on wire. Laying a wireless network would be much simpler than struggling with wire in difficult terrains. The technology technique in future.also promises to be eco friendly in comparison to today's technologies. Hence all in all wireless transmission of energy could be the key power transmission

\section{REFERENCES:}

[1].http://image.gsfc.nasa.gov/poetry/ask/q768.html

[2].http://en.wikipedia.org/wiki/Nikola_Tesla

[3].http://en.wikipedia.org/wiki/Wardenclyffe_Tower

[4].http://www.teslasociety.com/teslacoil.htm

[5]. http://www.WiTricity.com/

[6]. http://www.engadget.com/2013/06/19/intel-joins-a4wp/

[7]. http://www.bbc.co.uk/news/technology-23603751

[8].http://www.academia.edu/1807442/A_SEMINAR_REPOR

T_ON_WIRELESS_ELECTRICITY_WiTricity_

[9].http://www.tsgc.utexas.edu/tadp/1996/general/wpt.html

[10]. http://powerbyproxi.com/

[11]. Sagolsem Kripachariya Singh, T. S. Hasarmani, and R.

M. Holmukhe, 'Wireless Transmission of Electrical Power Overview of Recent Research \& Development, International Journal of Computer and Electrical Engineering, Vol.4, No.2, April 2012 